\title{
Relative Coprime Probability and Graph for Some Nonabelian Groups of Small Order and Their Associated Graph Properties
}

\author{
Nurfarah Zulkifli, Nor Muhainiah Mohd Ali* \\ Department of Mathematical Sciences, Faculty of Science, Universiti Teknologi Malaysia, 81310, UTM, Johor Bahru, Johor, Malaysia
}

Received August 20, 2021; Revised September 12, 2021; Accepted October 27, 2021

\section{Cite This Paper in the following Citation Styles}

(a): [1] Nurfarah Zulkifli, Nor Muhainiah Mohd Ali , "Relative Coprime Probability and Graph for Some Nonabelian Groups of Small Order and Their Associated Graph Properties," Mathematics and Statistics, Vol. 9, No. 6, pp. 859 - 866, 2021. DOI: 10.13189/ms.2021.090601.

(b): Nurfarah Zulkifli, Nor Muhainiah Mohd Ali (2021). Relative Coprime Probability and Graph for Some Nonabelian Groups of Small Order and Their Associated Graph Properties. Mathematics and Statistics, 9(6), 859 - 866. DOI: 10.13189/ms.2021.090601.

Copyright $\bigcirc 2021$ by authors, all rights reserved. Authors agree that this article remains permanently open access under the terms of the Creative Commons Attribution License 4.0 International License

\begin{abstract}
Let $G$ be a finite group. The probability that two selected elements $k$ from $K$ and $g$ from $G$ are chosen at random in a way that the greatest common divisor also known as gcd, of the order of $k$ and $g$, which is equal to one, is called as the relative coprime probability. Meanwhile, another definition states that the vertices or nodes are the elements of a group and two distinct vertices or nodes are adjacent if and only if their orders are coprime and any of them is in the subgroup of the group and this is called as the relative coprime graph. This research focuses on determining the relative coprime probability and graph for cyclic subgroups of some nonabelian groups of small order and their associated graph properties by referring to the definitions and theorems given by previous researchers. Besides, various results of the relative coprime probability for nonabelian groups of small order are obtained. As for the relative coprime graph, the result shows that the domination number for each group is one whereas the number of edges and the independence number for each group vary. Types of graphs that can be formed are either star graph, planar graph or complete $r$-partite subgraph depending on the order of the subgroup of a group.
\end{abstract}

Keywords Relative Coprime Probability, Relative Coprime Graph, Types of Graph, Domination Number, Independence Number

\section{Introduction}

In the study of the extension of coprime graph, Williams [1] was the first person to introduce the prime graph in 1981, which later, this study has attracted the attention of many researchers until they began to extend the prime graph to the coprime graph and it is defined in Definition 1 below.

\section{Definition 1: [2] Coprime Graph of a Group}

A graph whose vertices are elements of $G$ and there are edges between two distinct vertices $x$ and $y$ if and only if $(|x|,|y|)=1$ and it is called as a coprime graph of $G$ and is denoted as $\Gamma_{G}$. Note that $(|x|,|y|)=\operatorname{gcd}(|x|,|y|)$.

In their paper, interesting results have been obtained including the properties and the types of graphs. At the same time, the coprime graphs which have the end vertices are also classified. Later, an extension of the coprime graph has been made where Abd Rhani [3] introduced the relative coprime graph of a group $G$, denoted as $\Gamma_{\text {copr }}(K, G)$, where $K$ is a subgroup of $G$ and the definition is stated in Definition 11. In the paper, the author determined the types and some properties of the graph and the results are stated in Section 2 since the 
results will be used throughout this research. Recently, Zulkifli and Mohd Ali in [4] and [5] continued the research of coprime graph done by Ma et al. [2] but the scope of group was mainly on the nonabelian metabelian groups of order less than 24 and order 24, respectively. Both papers were targeted on finding the types of graphs, the domination number, the independence number, and the number of edges that can be obtained within a group.

Influenced by the research of the extension of coprime graph, Abd Rhani [3] decided to introduce a probability named the coprime probability of a group, denoted as $P_{c o p r}(G)$. It is defined that as the probability of a random pair of elements in a group $G$ is said to be coprime only happen when the greatest common divisor of the order of $x$ and $y$, where $x$ and $y$ are in $G$, which is equal to one. In [3], the results are limited where only the case of coprime probability for all $p$-groups and for some dihedral groups, $D_{n}$ where $n \geq 3$ and $n$ is odd are discussed. Later, Zulkifli and Mohd Ali [4] made an extensive research on the coprime probability in which the scope of the group is on the nonabelian metabelian groups of order less than 24 . The results showed that the coprime probability of dihedral groups or $p$-groups are the same when $G$ has the same order. The same method was applied in [5] where the authors targeted the same group as [4] but the scope was on the order 24. It was found that the results were varied.

Thus, in this paper, research on the coprime probability is continued further by introducing and determining the relative coprime probability for cyclic subgroups of some nonabelian groups of small order together with their graphs. The objective of this research is to introduce new definition and gives some propositions with the purpose of studying the uniqueness of the graphs and probabilities that can be obtained within a group.

Probability is one of the interesting topics of mathematics where it is always applied by statisticians to solve any uncertainties. Fortunately, probability has also been successfully applied in the field of group theory and one of the results can also be seen in this research where the relative coprime probability of a group is introduced and discussed in Section 3. At the same time, the study of coprime probabilities and its graphs can certainly benefit researchers in the field of group and graph theory for further study and investigation.

Hence, the relative coprime probability of a group is defined first and then it is determined for cyclic subgroups, $K$ of some nonabelian groups of small order. Later, the graphs for each $K$ in each group is identified along with their properties such as the types of graphs, the number of edges, the domination number, and the independence number.

Therefore, the first part of this paper is the introduction of this research whereas the second part provides the basic concepts such as definitions and theorems given by previous researchers which are very useful in this research.
Finally, the main results of this research are obtained and discussed in the third part of this paper and the overall research is summarized in the Conclusion section.

\section{Preliminaries on Groups and Graphs}

Some definitions in group and graph theory which are used in this research are provided in this section. This section starts by providing the definitions of some groups such as the dihedral groups, $p$-groups and quasi-dihedral groups.

\section{Definition 2: [6] Dihedral Group of Degree $n$}

The dihedral group, $D_{n}$ where its order is $2 n$ or equivalently to $\left|D_{n}\right|=2 n, \quad n \in \mathbb{Z} \quad$ and $n \geq 3$, symbolized as the set of symmetries of a regular $n$-gon. The dihedral group, $D_{n}$ is represented in the form of generators and relations, which it is given as follows:

$$
D_{n}=\left\langle a, b \mid a^{n}=e, b^{2}=e, a b=a^{-1} b\right\rangle .
$$

\section{Definition 3: [7] $p$-group}

Group $G$ is $p$-group if and only if the order of $G$ is a power of $p$, where $p$ is a prime number.

\section{Definition 4: [8] Quasi-dihedral Group}

The quasi-dihedral group, $Q_{D_{2^{n}}}$, in which the order of the group is $2^{n}$ where $n \geq 4$, is represented in the form of generators and relations and it is given as follows

$$
Q_{D_{2^{n}}}=\left\langle a, b \mid a^{2^{n-1}}=b^{2}=e, b a=a^{2^{n-2}-1} b\right\rangle .
$$

Next, some basic definitions in graph theory that will be used throughout this research including the star graph, planar graph, complete $r$-partite subgraph, the domination number, the independence number, and some other related theorems, are given in this section.

\section{Definition 5: [9] Start Graph}

A graph which comprises a single vertex with $n$ neighbours is called a star graph.

\section{Definition 6: [10] Planar Graph}

A planar graph is said to be a graph that can be drawn in a plane, in such a way that no two edges intersect except at the vertices. Else, it is said to be non-planar.

\section{Definition 7: [11] Complete $r$-partite Graph}

A complete $r$-partite graph is a $r$-partite graph 
having partition with the size of $n 1, n 2, \ldots, n r$ in a way that there are edges in every vertex in each partition to all vertices in the remaining partition.

\section{Definition 8: [10] Domination Number}

The dominating set $X \subseteq V(\Gamma)$ is a set where for each $y$ outside $X$, there exists $x \in X$ such that $y$ is adjacent to $x$. The domination number, $\gamma(\Gamma)$, is the minimum size of $X$.

\section{Definition 9: [10] Independence Number}

The independence number, denoted as $\alpha(\Gamma)$, is the number of vertices in the maximum independent set. The independent set is defined as a non-empty set $S$ of $V(\Gamma)$ whereby there is no adjacent between two elements of $S$ in $\Gamma$.

Theorem 1 [2] Let $K$ be a subgroup of a group $G$. Then $\Gamma_{\text {copr }}(K, G)$ is a star graph if and only if $G$ is a $p$-group, where $p$ is a prime number or $|K|=1$.

Theorem 2 [2] Let $K$ be a subgroup of a group $G$. Then $\Gamma_{\text {copr }}(K, G)$ is a planar graph if and only if $|K|=1$ or $|K|=2$ or $G$ is a $p$-group, where $p$ is a prime number.

Theorem 3 [2] Let $K$ be a subgroup of a group $G$. Let $\{e\}$ be a unique domination set or order 1 of $\Gamma_{\text {copr }}(K, G) . \quad$ Then $\quad \gamma\left(\Gamma_{\text {copr }}(K, G)\right)=1 \quad$ and $\operatorname{deg}(e)=|G|-1$.

Theorem 4 [2] Let $G$ be a group of order $p_{1}^{\delta_{1}} p_{2}^{\delta_{2}} \ldots p_{t}^{\delta_{t}}$ and $K$ be a subgroup of order $p_{1}^{\beta_{1}} p_{2}^{\beta_{2}} \ldots p_{s}^{\beta_{s}}$ where $\delta_{i}, \beta_{i}$ are positive integers, $p_{i}$ 's are prime numbers, $s \leq t$ and $0 \leq \beta_{i} \leq \delta_{i}$. Let $A_{i}$ be an independent set. Then $\alpha\left(\Gamma_{\text {copr }}(K, G)\right)=$ $\max \left\{\left|A_{i}\right|,|G \backslash K|\right\} \quad$ where $1 \leq i \leq t$.

\section{Main Results}

This section consists of two parts. The first part presents the relative coprime probability for some nonabelian groups of small order such as the dihedral groups, $p$-groups and quasi-dihedral groups of small order. Subsequently, the relative coprime graphs of the groups together with its properties are obtained in the second part of this section.

\subsection{The Relative Coprime Probability for Some Nonabelian Groups of Small Order}

Before proceeding with the outcomes that can be obtain in determining the relative coprime probability of the dihedral groups, $p$-groups and quasi-dihedral groups of small order, firstly, the relative coprime probability of a group is defined and given below.

\section{Definition 10: The Relative Coprime Probability of a Group}

Let $G$ be a finite group and $K$ be a subgroup of $G$. The relative coprime probability of $G$, denoted as $P_{c o p r}$, is stated as follows:

$$
P_{\text {copr }}(K, G)=\frac{|\{(k, g) \in K \times G \mid(|k|,|g|)=1\}|}{|K||G|} .
$$

Next, from Definition 10, the relative coprime probability for some nonabelian groups of small order is given.

3.1.1. Relative Coprime Probability for Dihedral Group of Order Six

Proposition 1 Let $G=D_{3}$. Then $G=\left\langle a, b \mid a^{3}=b^{2}=1, \quad b a b=a^{-1}\right\rangle$. Also, let $K$ be a cyclic subgroup of $G$. Then,

$$
P_{\text {copr }}(K, G)= \begin{cases}1, & \text { if } K=\langle e\rangle, \\ 3 / 4, & \text { if } K=\langle b\rangle, K=\langle a b\rangle, K=\left\langle a^{2} b\right\rangle, \\ 7 / 9, & \text { if } K=\langle a\rangle .\end{cases}
$$

\section{Proof}

Let $G=D_{3}=\left\{e, a, a^{2}, b, a b, a^{2} b\right\}$, where $|G|=6$ and the order of $\forall g \in G$ is $|e|=1, \quad|b|=|a b|=\left|a^{2} b\right|=2$, and $|a|=\left|a^{2}\right|=3$. Let $K$ be cyclic subgroups of $G$, then $|K|=1,2$ or 3 . Hence, the relative coprime probability is discussed.

Case 1: $|K|=1$

If $|K|=1, \quad$ then $K=\langle e\rangle . \quad$ Thus, $\forall g \in G$, $P_{\text {copr }}(K, G)=1$ since $(|e|,|g|)=1$.

Case 2: $|K|=2$

If $|K|=2$, then $K=\langle b\rangle, K=\langle a b\rangle$ or $K=\left\langle a^{2} b\right\rangle$ $K=\langle b\rangle=\{e, b\}, \quad\left(|e|,\left|g_{1}\right|\right)=1 \quad$ for $\quad$ all $\quad g_{1} \in G \quad$ and $\left(|b|,\left|g_{2}\right|\right)=1$ if $\left|g_{2}\right|=1$ or 3 , i.e. $g_{2}=e, a$ or $a^{2}$ 
Thus,

$$
\begin{aligned}
P_{c o p r}(K, G) & =\frac{\sum\left(e, g_{1}\right)+\sum\left(b, g_{2}\right)}{|K||G|} \\
& =\frac{6+3}{2(6)} \\
& =\frac{3}{4} .
\end{aligned}
$$

The same results are obtained for $K=\langle a b\rangle$ and $K=\left\langle a^{2} b\right\rangle$

Case 3: $|K|=3$

$$
\text { If }|K|=3, \quad \text { then } \quad K=\langle a\rangle=\left\{e, a, a^{2}\right\} \text {. }
$$

So,

$\left(|e|,\left|g_{1}\right|\right)=1 \quad$ for $\quad$ all $\quad g_{1} \in G, \quad$ and $\left(|a|,\left|g_{2}\right|\right)=\left(\left|a^{2}\right|,\left|g_{2}\right|\right)=1 \quad$ if $\quad\left|g_{2}\right|=1 \quad$ or $\quad 2$, i.e $g_{2}=e, b, a b$, or $a^{2} b$

Thus,

$$
\begin{aligned}
P_{\text {copr }}(K, G) & =\frac{\sum\left(e, g_{1}\right)+\sum\left(a, g_{2}\right)+\sum\left(a^{2}, g_{2}\right)}{|K||G|} \\
& =\frac{6+4+4}{3(6)} \\
& =\frac{7}{9} .
\end{aligned}
$$

The same steps can be applied to identify the relative coprime probability for cyclic subgroups of other dihedral groups of small order.

\subsubsection{Relative Coprime Probability for $p$-groups of Order Eight}

By the definition of $p$-groups where $G$ is a power of $p$ and $p$ is prime, one of the groups that is a $p$-groups is $D_{4}$., because $\left|D_{4}\right|=8=2^{3}$ and 2 is prime. Next, the relative coprime probability of $D_{4}$ is given.

Proposition 2 Let $G=D_{4}$. Then $G=\left\langle a, b \mid a^{4}=b^{2}=1, \quad b a b=a^{-1}\right\rangle$. Also, let $K$ be a cyclic subgroup of $G$. Then,

$$
P_{\text {copr }}(K, G)=\left\{\begin{aligned}
1,, & \text { if } K=\langle e\rangle, \\
9 / 16, & \text { if } K=\left\langle a^{2}\right\rangle, K=\langle b\rangle, K=\langle a b\rangle, K=\left\langle a^{2} b\right\rangle, \\
K & =\left\langle a^{3} b\right\rangle, \\
11 / 32, & \text { if } K=\langle a\rangle .
\end{aligned}\right.
$$

\section{Proof}

Let $G=D_{4}=\left\{e, a, a^{2}, a^{3}, b, a b, a^{2} b, a^{3} b\right\}, \quad$ where $|G|=8$ and the order of $\forall g \in G$ is $|e|=1$, $\left|a^{2}\right|=|b|=|a b|=\left|a^{2} b\right|=\left|a^{3} b\right|=2$, and $|a|=\left|a^{3}\right|=4$. Let $K$ be cyclic subgroups of $G$, then $|K|=1,2$ or 4 . Hence, the relative coprime probability of $D_{4}$ is stated below.

Case 1: $|K|=1$

If $|K|=1$, then $K=\langle e\rangle$. Thus, $\forall g \in G$, $P_{\text {copr }}(K, G)=1$ since $(|e|,|g|)=1$.

Case 2: $|K|=2$

If $|K|=2$, then $K=\left\langle a^{2}\right\rangle, K=\left\langle a^{2} b\right\rangle, K=\left\langle a^{3} b\right\rangle$, $K=\langle a b\rangle \quad$ or $\quad K=\langle b\rangle$. For $K=\left\langle a^{2}\right\rangle=\left\{e, a^{2}\right\}$, $\left(|e|,\left|g_{1}\right|\right)=1 \quad$ for all $g_{1} \in G$ and $\left(\left|a^{2}\right|,\left|g_{2}\right|\right)=1$ if $\left|g_{2}\right|$ $=1$, i.e. $g_{2}=e$.

Thus,

$$
\begin{aligned}
P_{\text {copr }}(K, G) & =\frac{\sum\left(e, g_{1}\right)+\sum\left(a^{2}, g_{2}\right)}{|K||G|} \\
& =\frac{8+1}{2(8)} \\
& =\frac{9}{16} .
\end{aligned}
$$

The same results are obtained for $K=\langle b\rangle$, $K=\langle a b\rangle, K=\left\langle a^{2} b\right\rangle$ or $K=\left\langle a^{3} b\right\rangle$

Case 3: $|K|=4$

If $|K|=4$, then $K=\langle a\rangle=\left\{e, a, a^{2}, a^{3}\right\}$. Let $k \in K$, then, $\left(|k=e|,\left|g_{1}\right|\right)=1$ for all $g_{1} \in G$, and $\left(|k \backslash e|,\left|g_{2}\right|\right)=$ 1 if $\left|g_{2}\right|=1$, i.e $g_{2}=e$.

Thus,

$$
\begin{aligned}
P_{c o p r}(K, G) & =\frac{\sum\left(e, g_{1}\right)+\sum\left(k \backslash e, g_{2}\right)}{|K||G|} \\
& =\frac{8+3}{4(8)} \\
& =\frac{11}{32} .
\end{aligned}
$$

The same steps are applied to obtain the relative coprime probability for cyclic subgroups of other $p$-groups of small order.

\subsubsection{Relative Coprime Probability for Quasi-dihedral} Group of Order Sixteen

Proposition 3 Let $G=Q_{D_{16}}$. Then $G=\left\langle a, b \mid a^{8}=b^{2}=1, \quad b a b=a^{3}\right\rangle$. Also, let $K$ be a 
cyclic subgroup of $G$. Then,

$$
P_{c o p r}(K, G)=\left\{\begin{aligned}
1, & \text { if } K=\langle e\rangle, \\
17 / 32, & \text { if } K=\left\langle a^{4}\right\rangle, K=\langle b\rangle, K=\left\langle a^{2} b\right\rangle, \\
K & =\left\langle a^{4} b\right\rangle, K=\left\langle a^{6} b\right\rangle, \\
19 / 64, & \text { if } K=\left\langle a^{2}\right\rangle, K=\langle a b\rangle, K=\left\langle a^{3} b\right\rangle, \\
23 / 128, & \text { if } K=\langle a\rangle,
\end{aligned}\right.
$$

Proof

Let

$G=Q_{D_{16}}=\left\{e, a, a^{2}, a^{3}, a^{4}, a^{5}, a^{6}, a^{7}, b, a b, a^{2} b, a^{3} b, a^{4} b\right.$, $\left.a^{5} b, a^{6} b, a^{7} b\right\}$ and the order of $\forall g \in G$ is $|e|=1$, $\left|a^{4}\right|=|b|=\left|a^{2} b\right|=\left|a^{4} b\right|=\left|a^{6} b\right|=2, \quad\left|a^{2}\right|=\left|a^{6}\right|=|a b|=\left|a^{3} b\right|=$ $\left|a^{5} b\right|=\left|a^{7} b\right|=4$, and $\left|a^{7}\right|=\left|a^{3}\right|=\left|a^{5}\right|=|a|=8$. Let $K$ be cyclic subgroups of $G$, then $|K|=1,2,4$ or 8 . Thus, the relative coprime probability is discussed.

Case 1: $|K|=1$

If $|K|=1$, then $K=\langle e\rangle$. Thus, $\forall g \in G, \quad P_{\text {copr }}(K, G)=1$ since $(|e|,|g|)=1$.

Case 2: $|K|=2$

If $|K|=2$, then $K=\left\langle a^{4}\right\rangle, K=\langle b\rangle, K=\left\langle a^{2} b\right\rangle, K=$ $\left\langle a^{4} b\right\rangle$ or $K=\left\langle a^{6} b\right\rangle$. For $K=\left\langle a^{4}\right\rangle=\left\{e, a^{4}\right\}$, $\left(|e|,\left|g_{1}\right|\right)=1$ for all $g_{1} \in G$ and $\left(\left|a^{4}\right|,\left|g_{2}\right|\right)=1$ if $\left|g_{2}\right|=1$ i.e $g_{2}=e$.

Thus,

$$
\begin{aligned}
P_{\text {copr }}(K, G) & =\frac{\sum\left(e, g_{1}\right)+\sum\left(a^{4}, g_{2}\right)}{|K||G|} \\
& =\frac{16+1}{2(16)} \\
& =\frac{17}{32} .
\end{aligned}
$$

The same results are obtained for $K=\langle b\rangle, K=\left\langle a^{2} b\right\rangle$, $K=\left\langle a^{4} b\right\rangle$ or $K=\left\langle a^{6} b\right\rangle$

Case 3: $|K|=4$

If $|K|=4$, then $K=\left\langle a^{2}\right\rangle, K=\langle a b\rangle$, and $K=\left\langle a^{3} b\right\rangle$. For $K=\left\langle a^{2}\right\rangle=\left\{e, a^{2}, a^{4}, a^{6}\right\}, \quad$ let $\quad k \in K \quad$ then, $\left(|k=e|,\left|g_{1}\right|\right)=1$ for all $g_{1} \in G$, and $\left(|k \backslash e|,\left|g_{2}\right|\right)=1$ if $\left|g_{2}\right|=1$, i.e. $\quad g_{2}=e$.

Thus,

$$
\begin{aligned}
P_{c o p r}(K, G)= & \frac{\sum\left(e, g_{1}\right)+\sum\left(k \backslash e, g_{2}\right)}{|K||G|} \\
& =\frac{16+3}{4(16)} \\
& =\frac{19}{64} .
\end{aligned}
$$

Case 4: $|K|=8$

If $|K|=8$, then $K=\langle a\rangle=\left\{e, a, a^{2}, a^{3}, a^{4}, a^{5}, a^{6}, a^{7}\right\}$.

Let $k \in K$ then, $\left(|k=e|,\left|g_{1}\right|\right)=1$ for all $g_{1} \in G$, and $\left(|k \backslash e|,\left|g_{2}\right|\right)=1$ if $\left|g_{2}\right|=1$, i.e $g_{2}=e$.

Thus,

$$
\begin{aligned}
P_{c o p r}(K, G) & =\frac{\sum\left(e, g_{1}\right)+\sum\left(k \backslash e, g_{2}\right)}{|K||G|} \\
& =\frac{16+7}{8(16)} \\
& =\frac{23}{128} .
\end{aligned}
$$

The same steps are applied to investigate the rest of the relative coprime probability for cyclic subgroups of other quasi-dihedral groups of small order.

Next, the relative coprime graphs of the three groups mentioned earlier are discussed.

\subsection{The Relative Coprime Graphs for Some Nonabelian Groups of Small Order}

As mentioned earlier, the relative coprime graph has been defined by Abd Rhani [3] and it is stated below.

\section{Definition 11: [2] Relative Coprime Graph of a Group}

Let $G$ be a finite group and $K$ be a subgroup of $G$. A graph whose vertices are elements of $G$ and two distinct vertices $x$ and $y$ are adjacent if and only if $(|x|,|y|)=1$ and any of $x$ or $y$ is in $K$ is known as the relative coprime graph of $G$ with respect to $K$, and it denoted as $\Gamma_{\text {copr }}(K, G)$,

The relative coprime graph together with their properties such as the domination number and the independence number are discussed next for dihedral groups, $p$-groups and quasi-dihedral groups of small order. These three groups are chosen to explain Definition 11 along with the properties that can be obtained from the graph of each group. 


\subsubsection{Relative Coprime Graph for Dihedral Groups of Order Six}

Let $G=D_{3}$, which is $G=\left\langle a, b \| a^{3}=b^{2}=1, b a b=a^{-1}\right\rangle=$ $\left\{e, a, a^{2}, b, a b, a^{2} b\right\}$ and for $K \leq G$, the relative coprime graphs are indicated below.

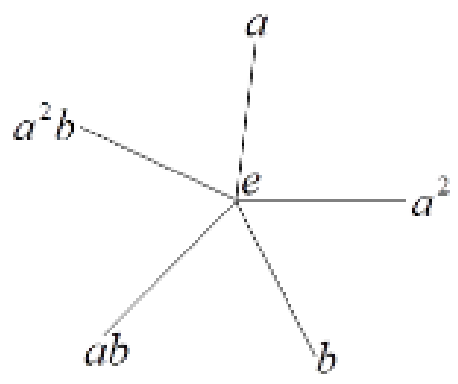

Figure 1. The relative coprime graph of $D_{3}$ with respect to $K$ for $|K|=1$

If $|K|=1$, then $K=\langle e\rangle$. From Figure 1, it can be seen that the total number of edges formed in this graph that connect to every vertex is 5 . The type of graphs obtained is a star graph and it is proven by Theorem 1. By Theorem 3, the domination number, $\gamma\left(\Gamma_{\text {copr }}(K, G)\right)=1$ and by Theorem 4, the largest independent set is $\left\{a, a^{2}, b, a b, a^{2} b\right\}$. Hence, the independence number, $\alpha\left(\Gamma_{\text {copr }}(K, G)\right)=5$.

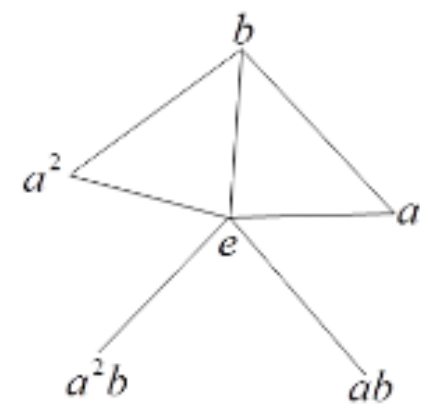

Figure 2. The relative coprime graph of $D_{3}$ with respect to $K$ for $|K|=2$

If $|K|=2$, then $K=\langle b\rangle, K=\langle a b\rangle$ or $K=\left\langle a^{2} b\right\rangle$. For $K=\langle b\rangle$, From Figure 2, it can be seen that the total number of edges formed in this graph that connect to every vertex is 7 . The type of graphs obtained is a planar graph and it is proven by Theorem 1. By Theorem 3, the domination number, $\gamma\left(\Gamma_{\text {copr }}(K, G)\right)=1$ and finally, by Theorem 4, the independence number, $\alpha\left(\Gamma_{\text {copr }}(K, G)\right)=4$ since the largest independent set for $\langle b\rangle$ is $\left\{a, a^{2}, a b, a^{2} b\right\}$. The same results are obtained for

$$
K=\langle a b\rangle \text { and } K=\left\langle a^{2} b\right\rangle .
$$

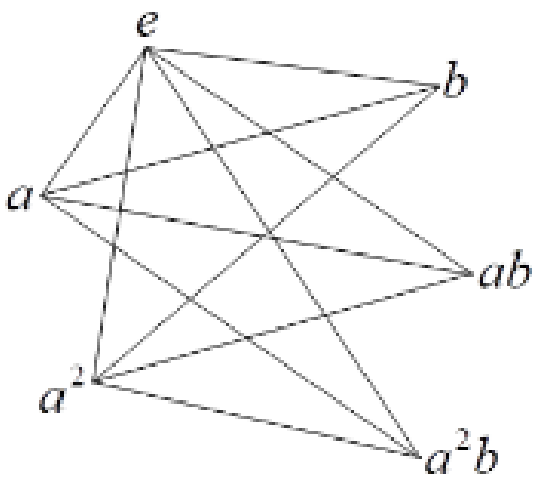

Figure 3. The relative coprime graph of $D_{3}$ with respect to $K$ for $|K|=3$

If $|K|=3$, then $K=\langle a\rangle$. From Figure 3, it can be seen that the total number of edges formed in this graph that connect to every vertex is 12 . The type of graphs obtained is a complete $r$-partite subgraph. By Theorem 3 , the domination number, $\gamma\left(\Gamma_{\text {copr }}(K, G)\right)=1$ and by Theorem 4 , the largest independent set is $\left\{b, a b, a^{2} b\right\}$. Hence, the independence number, $\alpha\left(\Gamma_{\text {copr }}(K, G)\right)=3$.

The same steps are applied to investigate the relative coprime graphs for cyclic subgroups of other dihedral groups of small.

\subsubsection{Relative Coprime Graph for $p$-groups of Order Eight}

As mentioned in 3.1.2, $\left|D_{4}\right|=8=2^{3}$ is also a $p$-group where $p=2$. In this subsection, $D_{4}$ is used as an example to discuss the relative coprime graph and its properties.

Let $G=D_{4}$, which is $G=\left\langle a, b \mid a^{4}=b^{2}=1, b a b=a^{-1}\right\rangle=$ $\left\{e, a, a^{2}, a^{3}, b, a b, a^{2} b, a^{3} b\right\}$ and for $K \leq G,|K|=1,2$ or 4. So, Figure 4 indicates the relative coprime graph.

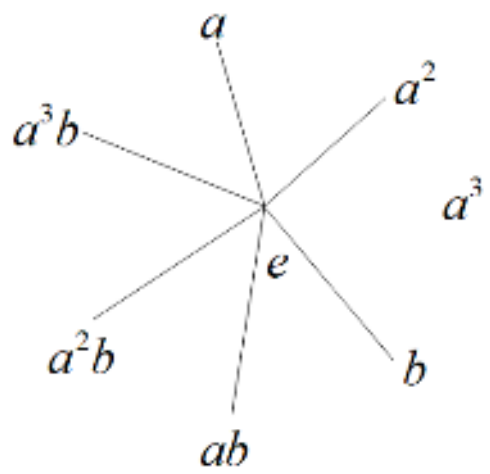

Figure 4. The relative coprime graph of $D_{4}$ with regard to $K$ 
From Figure 4, it can be seen that the total number of edges formed in this graph that connect to every vertex is 7. The type of graphs obtained is a star graph and it is proven by Theorem 1. In this graph, by Theorem 3, the domination number, $\gamma\left(\Gamma_{\text {copr }}(K, G)\right)=1$ and by Theorem 4 , the largest independence set is $\left\{a, a^{2}, a^{3}, b, a b, a^{2} b, a^{3} b\right\}$. Hence, the independence number, $\alpha\left(\Gamma_{\text {copr }}(K, G)\right)=7$.

The same steps are applied to investigate the relative coprime graphs for cyclic subgroups of other $p$-groups of small order.

\subsubsection{Relative Coprime Graph for Quasi-dihedral Groups of Order Sixteen}

Let $G=Q_{D_{16}}$, which is $G=\left\langle a, b \mid a^{8}=b^{2}=1, b a b=a^{3}\right\rangle=$ $\left\{e, a, a^{2}, a^{3}, a^{4}, a^{5}, a^{6}, a^{7}, b, a b, a^{2} b, a^{3} b, a^{4} b, a^{5} b, a^{6} b, a^{7} b\right\}$ and for $K \leq G,|K|=1,2,4$ or 8 . So, Figure 5 indicates the relative coprime graph.

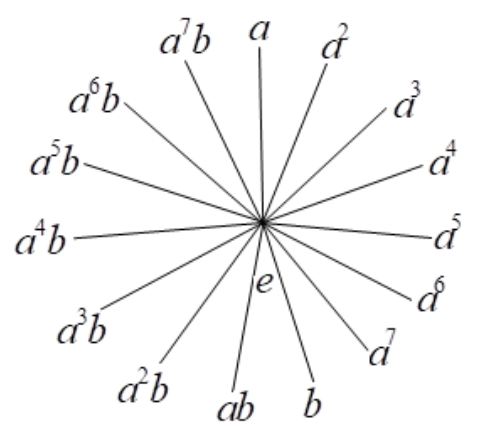

Figure 5. The relative coprime graph of $Q_{D_{16}}$ with respect to $K$

Therefore, it can be seen that the total number of edges formed in this graph that connect to every vertex is 15 . By Theorem 1, the graph is a star graph; by Theorem 3, the domination number, $\gamma\left(\Gamma_{\text {copr }}(K, G)\right)=1$; and finally, by Theorem 4, it can be concluded that the largest independent set is $\left\{a, a^{2}, a^{3}, a^{4}, a^{5}, a^{6}, a^{7}, b, a b, a^{2} b, a^{3} b, a^{4} b, a^{5} b, a^{6} b, a^{7} b\right\}$. Hence, the independence number, $\alpha\left(\Gamma_{\text {copr }}(K, G)\right)=15$.

\section{Conclusions}

The purpose of this research is to identify the relative coprime probability and graph along with the graph properties for some nonabelian groups which are the dihedral group of order 6, $p$-group of order 8 and quasi-dihedral groups of order 16. It can be concluded that the results of the relative coprime probability for each group mentioned before, vary. Simultaneously, the relative coprime graph together with its properties obtained a different result whereby for quasi-dihedral group, $Q_{D_{16}}$ and $p$-group, $D_{4}$, a star graph is obtained. For dihedral group of degree $n$, where $n$ is odd and $n \geq 3$, the types of graph formed in $D_{3}$ is a star graph for the case of $|K|=1$. When $|K|=2$, a planar graph is formed instead whereas if $|K|=3$, then the types of graph is a complete $r$-partite subgraph. Other properties such as the domination number for some nonabelian groups of order less than 24 is equal to one whereas the number of edges and the independence number for each group vary.

For further exploration on this research, other properties of the graphs can also be obtained such as the chromatic number, the diameter, the clique number, and the girth of a graph of each group. Besides, further extension on the relative coprime probability of a group can also be done by introducing a new probability which is the probability that two selected elements $k$ from $K$ and $g$ from $G$ are chosen at random when the greatest common divisor of the order of $k$ and $g$, is not equal to one, $(|k|,|g|) \neq 1$.

\section{Acknowledgements}

The first author would like to thank the Ministry of Higher Education (MOHE) for the financial support through MyBrainSc. The first and second author would like to express their gratitude to Universiti Teknologi Malaysia (UTM) for their total support.

\section{REFERENCES}

[1] J. Williams. Prime graph components of finite groups, Journal of Algebra, Vo.69, No.2, 487-513, 1981.

[2] X. Ma, H. Wei, L. Yang. The co-prime graph of a group, International Journal of Group Theory, Vol.3, No.3, 13-23, 2014.

[3] N. Abd Rhani. Some Extensions of the Commutativity Degree and the Relative Co-prime Graph of Some Finite Groups [Ph.D Thesis], Universiti Teknologi Malaysia, 2018.

[4] N. Zulkifli, N. M. Mohd Ali. Co-prime probability for nonabelian metabelian groups of order less than 24 and their related graphs, MATEMATIKA: Malaysian Journal of Industrial and Applied Mathematics, Vol.3, No.3, 357-369, 2019.

[5] N. Zulkifli, N. M. Mohd Ali. Co-prime probability for nonabelian metabelian groups of order 24 and their related graphs, Menemui Matematik (Discovering Mathematics), Vol.41, No.2, 68-79, 2019. 
[6] D. S. Dummit, R. M. Foote. Abstract Algebra, John Wiley and Son, USA, 2004.

[7] T.W. Judson. Abstract Algebra: Theory and Application, Orthogonal Publishing L3C, Michigan, 2015.

[8] S. Jennifer, S. Kathryn. On the structure of symmetric spaces of semidihedral groups, Involve, a Journal of Mathematics Vol.10, No 4, 665-676, 2017.
[9] C. Godsil, G. Royle. Algbebraic Graph Theory. 5th Ed, Springer, Boston New York, 2001.

[10] J. A. Bondy, U. S. A. Murty. Graphs Theory (Graduate Texts in Mathematics), Springer, New York, 2008.

[11] F. Harary. Graph Theory, Addison-Wesley, Philippines, 1969. 